\title{
Homocisteína en niños y adolescentes. Relación con historia familiar de enfermedad cardiovascular
}

\author{
Víctor Casanueva E, Ximena Cid Ca, Matilde Cancino M, \\ Laura Borzone $\mathrm{T}^{\mathrm{b}}$, Luis Cid $\mathrm{Sc}^{\mathrm{c}}$.
}

Serum homocysteine levels in children with and without a family history of cardiovascular disease

Background: Hyperhomocysteinemia is an independent cardiovascular risk factor that depends on folate and vitamin B12 nutrition. Aim: To measure homocysteine, folic acid and vitamin B12 serum levels in healthy children with and without a family history of cardiovascular disease. Subjects and methods: Forty children aged 6 to 15 years with a family history of cardiovascular disease, and 40 age and sex matched children without such history were studied. Serum homocysteine, folic acid and vitamin B12 were measured in a fasting blood sample. Homocysteine was measured by a fluorescence polarization immunoassay (FPIA), vitamin B12 by enzymatic microparticle assay, covered with intrinsic factor and folic acid by ionic capture, using commercial kits. Results: Children with family history of cardiovascular disease had higher homocysteine levels than their counterparts without family history $(7.9 \pm 3$ and $5.8 \pm 2 \mu \mathrm{mol} / \mathrm{l}$ respectively, $\mathrm{p}<0.03)$, but similar folic acid ( $5.2 \pm 1.8$ and $5.5 \pm 1.4 \mathrm{pg} / \mathrm{ml}$ respectively) and vitamin B12 levels $(431 \pm 213$ and $445 \pm 209 \mathrm{ng} / \mathrm{ml}$ respectively). There was a negative and significant correlation between homocysteine and folic acid and vitamin B12 levels. Conclusions: Children with a family history of cardiovascular disease have higher levels of serum homocysteine than those without such history, despite having similar levels of folic acid and vitamin B12 (Rev Méd Chile 2003; 131: 997-1002).

(Key Words: Cardiovascular diseases; Folic acid; Homocysteine; Risk factors; Vitamin B12)

Recibido el 13 de noviembre, 2002. Aceptado en versión corregida el 7 de julio, 2003. Trabajo financiado por la Dirección de Investigación № 99088018-10.

Departamento de Pediatría, Facultad de Medicina, Universidad de Concepción. Laboratorio Central, Hospital Clínico Regional, Servicio de Salud Concepción. Departamento de Estadística, Facultad de Ciencias Físicas y Matemáticas, Universidad de Concepción.

aBioquímico

becnólogo Médico

cPhD Estadística

Correspondencia a: Víctor Casanueva E. Departamento de Pediatría, Facultad de Medicina, Universidad de Concepción, Chile. Casilla 160-C. Fax: 41-254026. E mail: vcasanue@udec.cl 
Lo os factores de riesgo cardiovascular comienzan a temprana edad y es posible predecir los niveles de colesterol en el adulto a partir de los valores observados en la niñez ${ }^{1}$.

Los factores clásicos tales como dislipidemias e hipertensión arterial sólo explican dos tercios de los casos de infarto de miocardio ${ }^{2}$. El National Cholesterol Education Program recomienda utilizar el antecedente de enfermedad familiar cardiovascular precoz para el tamizaje de hipercolesterolemia en la niñez y adolescencia ${ }^{3}$. Sin embargo, no sólo la dislipidemia, que según algunos autores sería inespecífica y poco sensible, influye en el desarrollo del daño aterosclerótico vascular que se manifiesta clínicamente en el adulto.

Por esta razón se están investigando nuevos factores tales como el nivel sérico elevado de homocisteína, considerado como factor de riesgo independiente del metabolismo lipídico ${ }^{4}$.

La homocisteína es un aminoácido sulfurado intermediario en el metabolismo de la metionina, la cual es un importante donante de metilos para los procesos metabólicos incluyendo la formación del DNA. La metionina es aportada por la dieta y actúa en coordinación con el fosfato de piridoxal para eliminar los oxidantes residuales, que son capaces de dañar al endotelio vascular ${ }^{5}$. La alteración en el metabolismo de la metionina puede ocasionar hiperhomocisteinemia. Estudios epidemiológicos ${ }^{6}$ y de metaanálisis ${ }^{7}$ la han asociado a eventos cardiovasculares en el adulto tales como infarto de miocardio, accidente vascular cerebral y arteriopatía periférica.

En su génesis hay factores genéticos detectados en estudios de niños con parientes varones que han presentado muerte prematura de causa cardiovascular ${ }^{8}$. También hay factores nutricionales, tales como ingesta reducida de folatos o de vitamina B12. En ayunas la homocisteína sérica es remetilada a metionina teniendo los folatos y vitamina B12 como coenzimas; en cambio, en la etapa postprandial, la homocisteína sigue la vía eliminatoria de transulfuración a cisteína la cual depende exclusivamente de la vitamina $\mathrm{B}^{5}$.

Considerando que los estudios de niveles de homocisteína en niños son escasos, que en nuestro medio solamente se han publicado valores en población normal adulta ${ }^{9}$ y continuando con la línea de estudios de factores de riesgo cardiovascular en pediatría ${ }^{10}$, estudiamos la distribución de la concentración de homocisteína sérica en niños y adolescentes con y sin antecedente de historia familiar de enfermedad cardiovascular precoz, correlacionándolos además con los niveles de ácido fólico y vitamina B12.

\section{PACIENTES Y MÉTOdOS}

Sujetos. Este estudio, de tipo transversal, se realizó entre los meses de mayo de 1999 y junio de 2000, en 80 niños y adolescentes de ambos sexos, entre 6 y 15 años de edad los que se dividieron en dos grupos, según sus antecedentes familiares de enfermedad cardiovascular precoz; 1) con antecedentes $(n=40)$ hijos de padre, madre o abuelos que hubieran sufrido infarto 0 accidente vascular encefálico precoz $(\leq 55$ años de edad documentados a través de ficha clínica que consignara clínicamente y a través de exámenes correspondientes la presencia de dicha patología), detectados originalmente mediante una encuesta en la Escuela 765 de Curanilahue y elegidos aleatoriamente de un grupo de 57 de ellos; 2) sin antecedentes $(n=40)$ considerados como control, obtenidos aleatoriamente de una población de 432 niños de la misma escuela y pareados por sexo y edad.

El estudio fue aprobado por el Comité de Etica de la Facultad de Medicina de la Universidad de Concepción y por las Secretarías Regionales Ministeriales de Salud y Educación.

Evaluación: Se efectuó una evaluación médica a los niños, con examen físico completo: peso, talla, índice de masa corporal (IMC), presión arterial.

Laboratorio: A todos ellos, previa firma de consentimiento informado de los padres y ayuno de $12 \mathrm{~h}$, se les extrajo una muestra de $10 \mathrm{ml}$ de sangre venosa, sin anticoagulante. La sangre rotulada en el laboratorio del Hospital de Curanilahue, fue trasladada en un depósito con hielo y remitida al Laboratorio del Hospital Guillermo Grant Benavente, de Concepción, lugar en que se efectuaron las determinaciones, en un lapso no mayor de $2 \mathrm{~h}$ desde su extracción. La muestra se centrifugó a baja velocidad durante $15 \mathrm{~min}$, el suero se separó en alícuotas y se determinaron homocisteína, vitamina B12, ácido fólico, colesterol total (CT), colesterol-HDL (C-HDL), 
colesterol-LDL (C-LDL), triglicéridos (TG), creatinina, glicemia, proteína total, albúmina, globulina, índice albúmina/globulina, y transaminasas.

La homocisteína se midió mediante inmunoensayo de polarización de la fluorescencia (FPIA), en que la homocisteína unida (forma oxidada) se reduce a homocisteína libre y ésta se convierte enzimáticamente en S-adenosil-L-homocisteína (SAH). La homocisteína y sus formas como disulfuro mixto y unidas a proteínas presentes en la muestra, se reducen y forman homocisteína libre utilizando ditiotreitol (DTT). SAH y el trazador marcado con fluorescencia compiten por los sitios de unión de las moléculas de los anticuerpos monoclonales. La intensidad de la luz polarizada fluorescente se mide con sistema óptico FPIA. El equipo utilizado fue IMX de Abbot. La correlación de este método con el sistema HPLC, según especificaciones de Abbot, es de 0,989.

La vitamina B12 se determinó mediante el ensayo enzimático de micropartículas recubiertas de factor intrínseco, en el que se forma el conjugado B12: fosfatasa alcalina, que a su vez forma un complejo conjugado -factor intrínsecomicropartícula que es detectado mediante el sustrato fosfato de 4-metil-umbeliferona, siendo medida la tasa de producción del producto fluorescente por el dispositivo óptico MEIA.

El folato se midió mediante la técnica de captura iónica que utiliza un reactivo soluble de afinidad, compuesto de proteína de unión a folato (FBP) acoplado a anticuerpos monoclonales, los que a su vez son acoplados covalentemente a carboximetilamilosa (polianión). El folato es cuantificado midiendo la población de sitios sin ocupar unidos a la matriz usando un conjugado de ácido pteroico (un análogo del folato) y fosfatasa alcalina como molécula de generación de señal, finalmente se agrega el sustrato 4-metilumberefil fosfato y se mide el producto fluorescente por el dispositivo óptico MEIA. Tanto el folato como la vitamina B12 fueron determinados en equipo AxSYM con reactivos Abbot.

Los antecedentes familiares de enfermedades cardiovasculares se obtuvieron mediante encuestas y fichas clínicas. Los datos obtenidos fueron resumidos en formularios diseñados según los objetivos, los cuales fueron previamente validados.
Estadística. Los datos fueron resumidos en Tablas, los resultados se expresaron como promedio, desviación estándar e intervalos de confianza. Se utilizó la prueba t de Student para comparaciones entre los grupos, análisis univariado. Para medir correlaciones se utilizó el coeficiente de correlación de Pearson y análisis de regresión múltiple ${ }^{11}$. El análisis estadístico de los resultados y la comparación de los grupos estudiados, se efectuó mediante pruebas paramétricas y no paramétricas para experimentos controlados. Se realizaron estudios de varianza considerando diseños experimentales multifactoriales ${ }^{12}$.

Tamaño de la muestra. El tamaño de la muestra se calculó mediante la fórmula:

$$
\mathrm{n}=\frac{\mathrm{Z}^{2} \sigma^{2}}{\mathrm{E}^{2}}
$$

donde $\mathrm{Z}^{2}$ corresponde al cuadrado del percentil de la distribución normal estándar del nivel de confianza seleccionado, de modo que para 95\% este valor corresponde a $1,96^{2} ; S^{2}=\sigma^{2}$ varianza muestral de homocisteína 1,199, E= nivel de precisión de la estimación, corresponde a 0,368 umol/L precisión 7\%; $n=40$.

Encuesta dietaria. Se aplicó una encuesta dietaria mediante un cuestionario de frecuencia del consumo de alimentos por una Nutricionista del Consultorio de Salud anexo a la escuela en estudio.

Los niveles de vitamina B12 y folatos se compararon con los recomendados por la Dietary Reference Intakes (DRI) ${ }^{13}$.

\section{RESULTADOS}

Los datos antropométricos y exámenes de laboratorio clínico se presentan en la Tabla 1. Los escolares, se clasificaron en un grupo con antecedentes familiares cardiovasculares y otro sin ese antecedente.

No hubo diferencias significativas en los parámetros de peso, talla, ni IMC entre ambos grupos. La encuesta alimentaria reveló una ingesta calónica de proteínas, lípidos y glúcidos aceptable para la edad ya que están bajo control por una nutricionista. Ambos grupos presentaron niveles adecuados en hemograma, glicemia, proteínas y perfil hepático. 
Tabla 1. Características clínicas y de laboratorio en niños con y sin antecedentes familiares cardiovasculares

\begin{tabular}{|lll|}
\hline Parámetro & $\begin{array}{l}\text { Grupo A } \\
\text { Promedios y DS }\end{array}$ & $\begin{array}{l}\text { Grupo B } \\
\text { Promedios y DS }\end{array}$ \\
\hline Edad (años) & $10,3 \pm 2,5$ & $10,3 \pm 2,5$ \\
Indice masa corporal (m/Kg2) & $20,4 \pm 3,4$ & $18,8 \pm 3,6$ \\
Presión arterial sistólica (mmHg) & $110,7 \pm 10$ & $110,3 \pm 9,1$ \\
Presión arterial diastólica (mmHg) & $63,8 \pm 8$ & $63,6 \pm 6,4$ \\
Datos de laboratorio & $0,78 \pm 0,09$ & $0,75 \pm 0,12$ \\
Creatinemia (mg/dL) & $39 \pm 2$ & $38,8 \pm 2$ \\
Hematocrito (\%) & $80 \pm 10$ & $78,2 \pm 7,9$ \\
Glicemia (mg/dL) & & \\
\hline
\end{tabular}

$A=$ Niños con antecedentes familiares cardiovasculares. $B=$ Niños sin antecedentes familiares cardiovasculares. $D S=$ Desviación estándar

La ingestión de vitamina B12 fue aceptable de acuerdo a las normas de la National Dietary Allowance $^{13}$. En cuanto a los folatos, solamente un niño del grupo con antecedentes familiares cardiovasculares consumía menos de 80 ug/día, cantidad mínima aceptable para esa edad.

El perfil lipídico de ambos grupos se observa en la Tabla 2. En el grupo control no hubo niños con hipercolesterolemia. En el grupo con antecedentes familiares hubo 6 niños con colesterol sérico total sobre $200 \mathrm{mg} / \mathrm{dL}$. Los triglicéridos estuvieron significativamente aumentados en el grupo con antecedentes ( $p<0,05$ ).
El grupo con antecedentes de enfermedad cardiovascular familiar presentó un nivel de homocisteína significativamente superior al grupo control $(p<0,03)$ (Tabla 3$)$. El promedio de los niveles de folato y de vitamina B12 fue aceptable en ambos grupos.

Se observó una correlación negativa entre la homocisteína y la vitamina B12 y entre la homocisteína y el ácido fólico, la que fue: para los niños con antecedentes de $r=0,38$ y $r=0,40(p<0,05)$, y para los sin antecedentes de $r=0,12$ y $r=0,14(p$ $<0,05)$, respectivamente.

Tabla 2. Lípidos y lipoproteínas en el grupo control (sin antecedentes) y con antecedentes de enfermedad cardiovascular precoz (promedio, desviación estándar, mínimo, máximo, intervalo de confianza 95\%)

\begin{tabular}{|c|c|c|c|c|c|c|c|c|c|c|c|c|c|}
\hline \multirow{3}{*}{$\begin{array}{l}\begin{array}{l}\text { Parámetro } \\
\mathrm{mg} / \mathrm{dl}\end{array} \\
\text { CT }\end{array}$} & \multicolumn{6}{|c|}{ Sin antecedentes } & \multicolumn{7}{|c|}{ Con antecedentes } \\
\hline & \multirow{2}{*}{$\begin{array}{l}\text { Promedio } \\
158\end{array}$} & \multirow{2}{*}{$\begin{array}{l}\text { Ds } \\
24\end{array}$} & \multirow{2}{*}{$\begin{array}{l}\text { Mín } \\
97\end{array}$} & \multirow{2}{*}{$\begin{array}{l}\text { Máx } \\
203\end{array}$} & \multicolumn{2}{|c|}{$\begin{array}{c}\text { Int de conf. } \\
95 \%\end{array}$} & \multirow{2}{*}{$\begin{array}{l}\text { Promedio } \\
168\end{array}$} & \multirow{2}{*}{$\begin{array}{l}\text { Ds } \\
30\end{array}$} & \multirow{2}{*}{$\begin{array}{l}\text { Mín } \\
105\end{array}$} & \multirow{2}{*}{$\begin{array}{l}\text { Máx } \\
247\end{array}$} & \multicolumn{2}{|c|}{$\begin{array}{c}\text { Int de conf. } \\
\quad 95 \%\end{array}$} & \multirow{2}{*}{$\begin{array}{l}\mathrm{p} \\
\mathrm{NS}\end{array}$} \\
\hline & & & & & 150 & 166 & & & & & 158 & 178 & \\
\hline C-HDL & 51 & 10 & 28 & 69 & 48 & 54 & 51 & 9 & 38 & 71 & 48 & 54 & NS \\
\hline C-LDL & 92 & 18 & 50 & 133 & 86 & 98 & 97 & 27 & 40 & 149 & 89 & 106 & NS \\
\hline $\mathrm{TG}$ & 79 & 31 & 44 & 182 & 69 & 89 & $100^{*}$ & 59 & 36 & 316 & 81 & 119 & 0,05 \\
\hline
\end{tabular}

$\mathrm{CT}=$ Colesterol total. $\mathrm{C}$-HDL= Colesterol de alta densidad. $\mathrm{C}$-LDL= Colesterol de baja densidad. TG=Triglicéridos. ${ }^{*} \mathrm{p}<0,05$ promedio TG entre niños con y sin antecedente familiar de riesgo cardiovascular. Ds= Desviación estándar. Mín= Mínimo. Max= Máximo. Int de conf= Intervalo de confianza. 


\section{Tabla 3. H omocisteína, ácido fólico y vitamina B12 en el grupo control (sin antecedentes) y con antecedentes de enfermedad cardiovascular precoz (promedio, desviación estándar, mínimo, máximo, intervalo de confianza)}

\begin{tabular}{|c|c|c|c|c|c|c|c|c|c|c|c|c|c|}
\hline \multirow{3}{*}{$\begin{array}{l}\text { Parámetro } \\
\begin{array}{l}\text { Homocisteína } \\
\text { Umol/l }\end{array}\end{array}$} & \multicolumn{6}{|c|}{ Sin antecedentes } & \multicolumn{7}{|c|}{ Con antecedentes } \\
\hline & \multirow{2}{*}{$\begin{array}{l}\text { Promedio } \\
5,8\end{array}$} & \multirow{2}{*}{$\begin{array}{l}\text { Ds } \\
2\end{array}$} & \multirow{2}{*}{$\begin{array}{l}\text { Mín } \\
3\end{array}$} & \multirow{2}{*}{$\begin{array}{l}\text { Máx } \\
10,9\end{array}$} & \multicolumn{2}{|c|}{$\begin{array}{l}\text { Int de conf. } \\
\quad 95 \%\end{array}$} & \multirow{2}{*}{$\begin{array}{l}\text { Promedio } \\
7,2^{*}\end{array}$} & \multirow{2}{*}{$\begin{array}{l}\text { Ds } \\
3\end{array}$} & \multirow{2}{*}{$\begin{array}{l}\text { Mín } \\
3,2\end{array}$} & \multirow{2}{*}{$\begin{array}{l}\text { Máx } \\
19,9\end{array}$} & \multicolumn{2}{|c|}{$\begin{array}{c}\text { Int de conf. } \\
\quad 95 \%\end{array}$} & \multirow{2}{*}{$\begin{array}{l}\mathrm{p} \\
0,03\end{array}$} \\
\hline & & & & & 5,1 & 6,4 & & & & & 6,1 & 8,3 & \\
\hline $\begin{array}{l}\text { Acido fólico } \\
\mathrm{pg} / \mathrm{ml}\end{array}$ & 5,5 & 1,4 & 2,5 & 8,5 & 5 & 6 & 5,2 & 1,8 & 1,8 & 9,6 & 4,7 & 5,8 & NS \\
\hline $\begin{array}{l}\text { Vitamina B12 } \\
\mathrm{ng} / \mathrm{ml}\end{array}$ & 445 & 209 & 130 & 1.020 & 375 & 515 & 431 & 213 & 117 & 1.093 & 360 & 502 & NS \\
\hline
\end{tabular}

${ }_{p}^{*}<0,03$ Promedio homocisteína entre niños con y sin antecedente familiar de riesgo cardiovascular. DS= Desviación estándar. Mín= Mínimo. Máx= Máximo. Int de conf= Intervalo de confianza.

\section{Discusión}

Se encontró una diferencia significativa, con niveles superiores de homocisteína en los niños que presentaban antecedente familiar de enfermedad cardiovascular precoz y una correlación negativa entre homocisteína con ácido fólico y vitamina B12.

Nuestros niveles de homocisteína observados en niños y adolescentes fueron similares a los encontrados en niños alemanes ${ }^{14}$, belgas ${ }^{15}$, norteamericanos ${ }^{1}$ y nonuegos ${ }^{8}$. Tal como en esos estudios, en general el promedio equivale a la mitad del de los adultos, hecho que también fue observado al comparar nuestros valores con los de adultos chilenos ${ }^{9}$, confirmándose así que estos niveles aumentan con la edad.

Al comparar los diversos estudios hemos encontrado sólo uno que utilizó la misma técnica empleada por nosotros y cuyos resultados fueron similares ${ }^{16}$. Los folatos obtenidos en nuestros niños estuvieron en rangos normales, tal como en los estudios $\mathrm{CATCH}^{17}$ (Child and Adolescent Trial for Cardiovascular Health) y belga ${ }^{15}$. La correlación negativa entre la homocisteína, folatos y vitamina B12 se explica porque la remetilación de la homocisteína depende de esas vitaminas para evitar su acumulación y derivar a metionina.

El ayuno previo a la determinación de homocisteína sérica es importante como se menciona en la introducción. Los estudios $\mathrm{CATCH}^{17}$ y de niños noruegos ${ }^{8}$ no fueron realizados en niños en ayunas, por lo tanto su relación con folatos es débil y no concluyente.
El CT no mostró diferencia significativa entre los niños con y sin antecedentes de cardiopatía precoz, confirmando así que este parámetro es inespecífico y poco sensible ${ }^{1}$. En un estudio de 15 países los niños noruegos tenían los niveles séricos de colesterol más elevados después de Finlandia, pero Noruega ocupa el cuarto lugar en la mortalidad cardiovascular. Esto apoya la hipótesis que la relación entre los lípidos séricos del niño y la enfermedad cardiovascular del adulto debe estar influida además por otros factores, tales como la homocisteína ${ }^{18}$.

Este análisis teórico de la importancia de la homocisteína en la génesis de la aterosclerosis ya en la niñez, tiene aplicaciones prácticas en clínica. Una ventaja de contrarrestar la hiperhomocisteinemia, mediante el consumo de vegetales y cereales enriquecidos con folato, es que toda la población se protege contra el inicio de la aterosclerosis en la niñez y su desarrollo en el adulto, en especial si hay otros factores de riesgo cardiovascular.

En conclusión, se encontró una diferencia significativa, con niveles superiores de homocisteína en los niños que portaban el antecedente familiar de enfermedad cardiovascular precoz, junto a una correlación negativa entre la homocisteína con ácido fólico y homocisteína con vitamina B12. Los valores son una primera alerta para considerar a este parámetro en futuros estudios de factores de riesgo cardiovascular. 


\section{REFERENCIAS}

1. Greenlund K, Srinivasan S, Xu J, Dalferes E, Myers L. Plasma Homocysteine Distribution and its Association with Parental History of Coronary Artery Disease in Black and White Children. The Bogalusa Heart Study. Circulation 1999; 2144-9.

2. Canri C, Constans J, Parrot F, Skopinski S, Cipriano C. Homocysteinemie: Rôle en Pathologie Vasculaire. Presse Med 2000; 29: 737-41.

3. National Cholesterol Education Program Report of the Expert Panel on Blood Cholesterol Levels in Children and Adolescents. Pediatrics 1992; 89 (suppl): 525-84.

4. Mc CuLy K. Homocysteine, Folate, Vitamin B6 and Cardiovascular Disease. JAMA 1998; 279: 392-3.

5. Brattstrom L, Wilcken D. Homocysteine and cardiovascular disease cause or effect? Am J Clin Nutr 2000; 72: 315-23.

6. Eikelboom J, Lonm E, Genest J, Hankey G, Yusuf S. Homocysteine and Cardiovascular Disease. A critical Review of the Epidemiologic Evidence. Ann Inter Med 1999; 131: 363-75.

7. WALD DS, LAW M, MorRIS JK. Homocysteine and cardiovascular disease: evidence and casualty from a meta analysis. BMJ 2002; 325: 1202-17.

8. Tonstad S, Refsum H, SivertSen M, Christophersen B, OSE L Relation of total homocysteine and lipid levels in children to premature cardiovascular death in male relatives. Pediatr Res 1996; 40: 47-52.TP

9. Bunout D, Petermann M, de la Maza P, Kauffmann R, Suazo M, Hirsch S. Niveles de homocisteína en adultos sanos chilenos. Rev Méd Chile 1998; 126: 905-10.
10. Casanueva V, Cid X, Chiang MT, Román R, Milos C, Reyes M et al. Perfil lipídico y prevalencia de dislipidemias en 2.102 niños y adolescentes de Concepción. Rev Méd Chile 1996; 124: 1453-61.

11. Draper N, Smith M. Applied Regression Analysis. $2^{\text {nd }}$ Edition. 1997. Wiley and Sons. New York.

12. Montgomery A. Design and Analysis of Experiments. 1997. $4^{\text {th }}$ Edition Wiley and Sons, New York.

13. Monsen E. Dietary Reference Intakes for the Antioxidant Nutrients. J Am Diet Ass 2000; 100: 641-6.

14. Rauh M, Verwield S, KNerR I, DorR H, SONMichsen A, KaletzKo B. Homocysteine concentrations in a German cohort of 500 individuals. Reference range and determinants of plasma level in healthy children and their parents. Amino Acids 2001; 20: 409-18.

15. De Laet C, Wautrecht J, Brasseur D, Dramaix M, Boeynaems J. Plasma homocysteine concentration in a Belgian school age population. Am J Clin Nutr 1999; 69: 968-72.

16. Laskwska-Kuta T, Szymczak E, Radomyska B. Serum homocysteine and lipoprotein (a) concentrations in hypercholesterolemic and normocholesterolemic children. Clin Pediatr 2001; 40: 149-54.

17. Osganian S, Stampfer M, Spegelman D, Rimm E, Cutter J, Feldman H. Distribution of and Factors Associated Serum Homocysteine Levels in Children. Child and Adolescent Trial for Cardiovascular Health (CATCH) JAMA 1999; 281: 1189-96.

18. Wiwams C, Wynder E. Cardiovascular risk factors in children from 15 countries. Cardiovascular Risk Factors 1992; 2: 45-55. 